\title{
Peak perihemorrhagic edema correlates with functional outcome in intracerebral hemorrhage
}

Bastian Volbers, MD, Antje Giede-Jeppe, MD, Stefan T. Gerner, MD, Jochen A. Sembill, MD, Joji B. Kuramatsu, MD, Stefan Lang, MD, Hannes Lücking, MD, Dimitre Staykov, MD, and

Hagen B. Huttner, MD, PhD

\section{Correspondence}

Dr. Volbers

bastian.volbers@gmx.net

Neurology ${ }^{\circ}$ 2018;90:e1005-e1012. doi:10.1212/WNL.0000000000005167

\begin{abstract}
\section{Objective}

To evaluate the association of perihemorrhagic edema (PHE) evolution and peak edema extent with day 90 functional outcome in patients with intracerebral hemorrhage (ICH) and identify pathophysiologic factors influencing edema evolution.
\end{abstract}

\section{Methods}

This retrospective cohort study included patients with spontaneous supratentorial ICH between January 2006 and January 2014. ICH and PHE volumes were studied using a validated semiautomatic volumetric algorithm. Multivariable logistic regression and propensity score matching (PSM) accounting for age, ICH volume, and location were used for assessing measures associated with functional outcome and PHE evolution. Clinical outcome on day 90 was assessed using the modified Rankin Scale (0-3 = favorable, 4-6 = poor).

\section{Results}

A total of 292 patients were included. Median age was 70 years (interquartile range [IQR] 62-78), median ICH volume on admission $17.7 \mathrm{~mL}$ (IQR 7.9-40.2). Besides established factors for functional outcome, i.e., ICH volume and location, age, intraventricular hemorrhage, and NIH Stroke Scale score on admission, multivariable logistic regression revealed peak PHE volume (odds ratio [OR] 0.984 [95\% confidence interval (CI) 0.973-0.994]) as an independent predictor of day 90 outcome. Peak PHE volume was independently associated with initial PHE increase up to day 3 (OR 1.060 [95\% CI 1.018-1.103]) and neutrophil to lymphocyte ratio on day 6 (OR 1.236 [95\% CI 1.034-1.477; PSM cohort, $\mathrm{n}=124]$ ). Initial PHE increase (PSM cohort, $\mathrm{n}=224$ ) was independently related to hematoma expansion (OR 3.647 [95\% CI 1.533-8.679]) and fever burden on days 2-3 (OR 1.456 [95\% CI $1.103-1.920])$.

\section{Conclusion}

Our findings suggest that peak PHE volume represents an independent predictor of functional outcome after ICH. Inflammatory processes and hematoma expansion seem to be involved in PHE evolution and may represent important treatment targets. 


\section{Glossary}

$\mathbf{C I}=$ confidence interval; $\mathbf{I C H}=$ intracerebral hemorrhage; $\mathbf{I C P}=$ intracranial pressure; $\mathbf{I Q R}=$ interquartile range; IVH = intraventricular hemorrhage; mRS = modified Rankin Scale; NIHSS = NIH Stroke Scale; NLR = neutrophil to lymphocyte ratio; $\mathbf{P H E}=$ perihemorrhagic edema; $\mathbf{P S M}=$ propensity score-matched; $\mathbf{O R}=$ odds ratio; $\mathbf{R O C}=$ receiver operating characteristic.

In intracerebral hemorrhage (ICH), there are many detrimental processes and secondary damage cascades, including inflammation and degradation of heme products, initiated immediately after ICH onset. They are hypothesized to contribute to the development of perihemorrhagic edema (PHE), which is suggested to serve as a radiologic marker for secondary injury following $\mathrm{ICH} .{ }^{1-4}$ PHE volume may evolve within a period of 8-12 days after ictus and may exceed the initial lesion volume by more than $150 \%,{ }^{3}$ possibly causing clinical deterioration or even herniation. ${ }^{5}$ Thus, PHE evolution and notably peak PHE volume may represent important outcome measures and treatment targets not only in the (hyper-)acute phase of ICH while established prognostic outcome measures (e.g., initial hematoma volume or age ${ }^{1}$ ) are rather inaccessible to a treatment or show limited treatment options. ${ }^{1,6-8}$ Specifically, several research groups reported on an association of early PHE evolution up to 24-72 hours after ICH onset with functional outcome. ${ }^{9-12}$ However, as PHE volume may peak 8-12 days after onset, analyzing PHE evolution merely up to 72 hours after onset may yield limited results. In the present study, we correlated peak PHE volume with long-term outcome in $\mathrm{ICH}$ patients. Furthermore, we assessed factors associated with increased PHE evolution.

\section{Methods}

\section{Patients}

Our study retrospectively included patients with spontaneous supratentorial ICH who were admitted between January 2006 and January 2014 from our prospectively organized institutional database. We defined ICH as spontaneous if exclusively related to hypertension or amyloid angiopathy. Patients with secondary ICH were excluded. Inclusion criteria were at least 2 consecutive CT scans over a period of at least 72 hours, no withdrawal of care within 24 hours after admission, an available modified Rankin Scale score (mRS) on day 90 , and a baseline $\mathrm{mRS}$ of $<4$. Furthermore, we excluded patients with surgical hematoma evacuation.

\section{Standard protocol approvals, registrations, and patient consents}

The study was approved by our institutional ethics committee.

\section{Clinical data}

We obtained age, ${ }^{1}$ mRS before symptom onset, NIH Stroke Scale score (NIHSS) on admission, ${ }^{1}$ and other outcome predicting measures in ICH such as fever burden (defined as number of days with peak temperature $\geq 38^{\circ} \mathrm{C}$ ) up to day $12^{13}$ and serum inflammatory measures ${ }^{14}$ including lymphocytes, neutrophils, and neutrophil to lymphocyte ratio (NLR; neutrophil count divided by lymphocyte count) on admission and days 4 and 6 as a marker for systemic inflammation also relevant for cardiovascular outcomes ${ }^{15}$ and elevated intracranial pressure (ICP) burden (number of episodes with ICP $>20 \mathrm{~mm} \mathrm{Hg}$ lasting more than 5 minutes or with patients presenting with symptoms related to elevated ICP [deterioration of consciousness, anisocoria]) up to day 12 from medical records. We used standardized mailed questionnaires for the assessment of functional status before symptom onset and comorbidities if those data were not obtained during hospital stay. Time between symptom onset and admission was assessed as an important predictor of hematoma expansion. ${ }^{16}$ The day of admission was defined as day 1 .

\section{Neuroimaging and volumetric assessment of PHE and ICH}

A fourth-generation CT scanner (Somatom 64 or Somatom AS+, Siemens Healthcare, Erlangen) was used for CT scans. Each scan consisted of either a multislice spiral CT dataset, 10-12 slices of $4.8 \mathrm{~mm}$ thickness for the skull base and 10-12 slices of $7.2 \mathrm{~mm}$ thickness for the cerebrum (Somatom 64), or 22-25 slices of $4.8 \mathrm{~mm}$ thickness for the entire brain (Somatom AS+) using the orbitomeatal plane. We used a validated semiautomatic volumetric algorithm as previously described ${ }^{17}$ for assessment of ICH and PHE volume. For better comparison, we merged different time points of $\mathrm{CT}$ scans to time clusters (days 1, 2-3, 4-6, 7-9, 10-12). Initial PHE growth from baseline to 72 hours $^{9-12,18}$ (defined as absolute difference between PHE volume $[\mathrm{mL}]$ on day 2-3 and admission), intraventricular hemorrhage (IVH), and hematoma volume on admission ${ }^{19}$ as well as peak PHE volume and secondary hematoma enlargement (hematoma expansion) volume were recorded. Peak PHE volume was defined as the maximum volume measured in any of the available CT scans. In the context of accuracy of measurements, ${ }^{20,21}$ a hematoma volume increase $\geq 5 \mathrm{~mL}$ between $2 \mathrm{CT}$ scans was defined as hematoma expansion. ${ }^{7}$

\section{Outcome}

We assessed functional outcome on day 90 using the mRS either by mailed standardized questionnaires or phone interviews (performed by trained and certified physicians) with patients or their next of kin if the questionnaire was not returned within 4 weeks. ${ }^{22}$ In cases of insufficient data retrieval, we contacted primary care physicians. As primary outcome variable, $\mathrm{mRS}$ was dichotomized as favorable outcome (mRS 0-3) or poor outcome (mRS 4-6). As secondary 
outcome measures, we analyzed the correlation of clinical characteristics and inflammatory measures with peak $\mathrm{PHE}$ volume and early PHE growth from baseline up to day 3 (72 hours). Patients were dichotomized according to median peak $\mathrm{PHE}$ volumes and median early PHE increase volumes.

\section{Statistics}

We performed statistical analyses using the IBM SPSS Statistics 21 software package (IBM Corporation, Armonk, NY) and R 2.12 .0 (r-project.org). The significance level was set at $\alpha=0.05$ and statistical tests were 2 -sided. Missing data regarding basic characteristics, neuroimaging, or outcome led to exclusion of patients. We used the Kolmogorov-Smirnov test to evaluate the distribution of data. Data were presented as mean \pm SD (statistical test: Student $t$ test), or as median and interquartile range (IQR, statistical test: Mann-Whitney $U$ test), as appropriate. We used Pearson $\chi^{2}$ or Fisher exact test to compare frequency distributions of categorized variables between dichotomized patient groups: (1) mRS 0-3 and 4-6, (2) patients with peak PHE volume smaller and larger than the median peak PHE volume, and (3) patients with an early PHE increase smaller and larger than the median increase of the included cohort (median split). For both groups 2 and 3, we performed a separate additional propensity score matching using the entire cohort $(\mathrm{n}=292)$ based on logistic regression as estimation algorithm (nearest neighbor matching, ratio $1: 1$, caliper $=0.1$ ) accounting for age, hematoma volume, and hematoma location (basal ganglia vs lobar) and calculated abovementioned univariate testing as well as additional logistic regression. To identify measures independently associated with outcome and peak PHE volume, we calculated stepwise backward inclusion multivariate logistic regression models including clinically meaningful measures showing a statistical trend $(p<0.1)$ in univariate testing of the entire cohort. As hematoma location (lobar vs basal ganglia) was demonstrated previously to represent an important outcome associated factor ${ }^{23}$ with a suggested interaction with hematoma volume, ${ }^{24}$ we also included the interaction term of both factors in our multivariate logistic regression model. Because not all patients had regular CT scans up to day 10-12 based on inclusion criteria, we performed an additional sensitivity analysis including only patients with at least 4 CT scans up to day 9. To explore associations of PHE evolution with outcome, we additionally performed receiver operating characteristic (ROC) curve analyses.

\section{Results}

\section{Cohort}

A total of 875 patients were screened. We excluded 154 patients who received early withdrawal of care, 53 patients because of surgery (hematoma evacuation) prior to control scan, and 140 patients who had only 1 CT scan. A further 38 patients were excluded due to insufficient datasets, 143 due to infratentorial hemorrhage, and 55 due to a $\mathrm{mRS}$ prior to the current $\mathrm{ICH}$ of $>3$. This led to 292 patients (161 male [55\%]) who met inclusion criteria. A total of 121 (41\%) patients presented with lobar hemorrhage and 171 (59\%) with basal ganglia hemorrhage. A total of 159 patients (54\%) showed an additional IVH. Median age was 70 years (IQR 62-78), median ICH volume on admission $17.7 \mathrm{~mL}$ (IQR 7.9-40.2), and median peak ICH volume 22.5 mL (IQR 8.9-46.4). Median PHE volume on admission was $16.3 \mathrm{~mL}$ (IQR 9.1-29.2) and increased to a median peak PHE volume of $37.5 \mathrm{~mL}$ (IQR 19.0-60.6) over a median period of 7 days (IQR 3-12). Median NIHSS on admission was 13 (IQR 6-21), median mRS on day 90 was 4 (IQR 3 to 5). Time between symptom onset and admission did not differ between patients with and without hematoma expansion up to day 3 (4.2 hours [IQR 2.1-9.0] and 5.0 hours [IQR 2.5-11.9], respectively, $p=0.234$ ).

\section{Factors influencing outcome in ICH patients}

A total of 107 patients (37\%) had a favorable outcome on day 90. Detailed characteristics of both outcome groups are shown in table 1 and table e-1 (links.lww.com/WNL/A259). Besides established prognostic factors such as ICH volume, location, age, and IVH, frequency of elevated ICP, fever burden, neutrophil to lymphocytes count on day 6, and peak PHE extent differed between both outcome groups in univariate analysis. Lymphocytes, neutrophils, and NLR on admission and day 4 did not differ between the outcome groups (data not shown). Multivariable logistic regression (table 2) revealed only peak $\mathrm{PHE}$ (odds ratio[OR]/Exp[B] 0.984 for each $\mathrm{mL}$ of $\mathrm{PHE}$ ) as independent prognostic factor in $\mathrm{ICH}$ patients besides the abovementioned and established measures. ${ }^{25}$ Peak PHE volume also remained the only independent outcome predictor in our sensitivity analysis including only patients with at least 4 CT scans up to day $9(\mathrm{n}=218)$ with a comparable OR (OR 0.985 [95\% confidence interval (CI) 0.973-0.997], $p=0.012$ ) besides and adjusted for the abovementioned factors.

Figure 1 illustrates PHE evolution in patients with favorable and poor outcome: patients with poor outcome showed an immense PHE increase during the first 72 hours after ICH onset compared to patients with a favorable outcome, and $\mathrm{PHE}$ increase continued to be less pronounced up to day 10-12.

\section{Receiver operating characteristic analysis}

ROC analysis (figure e-1, links.lww.com/WNL/A258) confirmed a good predictive value of peak PHE regarding favorable outcome, especially compared to the predictive value of initial ICH volume and the well-established $\mathrm{ICH}$ score. ${ }^{25} \mathrm{~A}$ cutoff value of $26.8 \mathrm{~mL}$ peak PHE volume was identified with a sensitivity of 0.54 and a specificity of 0.78 (Youden Index $0.326)$ to distinguish best between patients with a favorable and a poor outcome.

\section{Measures influencing peak PHE volume}

Given that peak PHE extent was identified as an independent predictor of long-term outcome, we studied factors influencing peak PHE volume. As demonstrated in table 3, in univariate analyses various measures including younger age, location of hematoma, IVH, hematoma volume, initial PHE increase up to day 3 , fever burden, and reduced lymphocyte count on day 4-6 as well as increased NLR on day 6 were 
Table 1 Clinical characteristics in favorable and poor outcome groups

\begin{tabular}{|c|c|c|c|}
\hline Spontaneous ICH ( $n=292)$ & $\begin{array}{l}\text { Favorable outcome } \\
\text { (mRS 0-3; } n=107 \text { ) }\end{array}$ & $\begin{array}{l}\text { Poor outcome } \\
\text { (mRS 4-6; } n=185 \text { ) }\end{array}$ & $p$ Value \\
\hline Age, y, median (IQR) & $69(59-76)$ & $72(64-79)$ & $0.007^{\mathrm{a}}$ \\
\hline Male sex, n (\%) & $57(53)$ & $104(56)$ & $0.714^{b}$ \\
\hline NIHSS on admission, median (IQR) & $5(2-11)$ & $16(11-35)$ & $<0.001^{\mathrm{a}}$ \\
\hline Baseline mRS, median (IQR) & $1(0-2)$ & $1(0-2)$ & $0.003^{a}$ \\
\hline \multicolumn{4}{|l|}{ Medication on admission, $\mathrm{n}(\%)$} \\
\hline Platelet aggregation inhibitors & $30(28)$ & $58(31)$ & $0.598^{\mathrm{b}}$ \\
\hline VKA & $16(15)$ & $34(18)$ & $0.521^{\mathrm{b}}$ \\
\hline Statin & $28(26)$ & $36(20)$ & $0.189^{b}$ \\
\hline Location lobar, $\mathrm{n}(\%)$ & $56(52)$ & $65(35)$ & $0.004^{b}$ \\
\hline Intraventricular hemorrhage, $\mathrm{n}(\%)$ & $38(36)$ & $121(65)$ & $<0.001^{\mathrm{b}}$ \\
\hline Hematoma volume on admission, $\mathrm{mL}$, median (IQR) & $10.6(4.9-25.6)$ & $21.4(11.2-44.4)$ & $<0.001^{\mathrm{a}}$ \\
\hline Hematoma expansion $>5 \mathrm{~mL}, \mathrm{n}(\%)$ & $15(14)$ & $46(25)$ & $0.028^{b}$ \\
\hline Perihemorrhagic edema volume on admission, $\mathrm{mL}$, median (IQR) & $13.7(5.7-25.9)$ & $18.5(10.9-34.0)$ & $<0.001^{\mathrm{a}}$ \\
\hline Perihemorrhagic edema volume increase up to day $3, \mathrm{~mL}$, median (IQR) & $1.97(-0.04$ to 6.5$)$ & $5.4(-0.3$ to 12.7$)$ & $0.028^{a}$ \\
\hline Peak perihemorrhagic edema volume, $\mathrm{mL}$, median (IQR) & $23.8(9-45.3)$ & $42.6(28.1-67.4)$ & $<0.001^{a}$ \\
\hline Intracranial pressure burden up to day 12 , median (IQR) & $0(0)$ & $0(0-5)$ & $<0.001^{\mathrm{a}}$ \\
\hline Fever burden up to day 12 , median (IQR) & $1(0-5)$ & $3(1-7)$ & $<0.001^{\mathrm{a}}$ \\
\hline Lymphocytes day $6,10^{9} / \mathrm{L}$, median (IQR) & $1.4(1.0-1.8)$ & $1.1(0.8-1.4)$ & $<0.001^{a}$ \\
\hline Neutrophil-to-lymphocyte ratio day 6 , median (IQR) & $4.0(2.9-5.4)$ & $4.61(3.2-6.7)$ & $0.005^{a}$ \\
\hline In-hospital mortality, n (\%) & $0(0)$ & $22(12)$ & $<0.001^{\mathrm{b}}$ \\
\hline mRS discharge, median (IQR) & $3(2-4)$ & $5(5-5)$ & $<0.001^{a}$ \\
\hline mRS day 90 , median (IQR) & $2(2-3)$ & $5(4-6)$ & $<0.001^{\mathrm{a}}$ \\
\hline $\begin{array}{l}\text { Abbreviations: ICH = intracerebral hemorrhage; IQR = interquartile range; NIHS } \\
\text { a Wilcoxon rank-sum test. } \\
{ }^{\circ} \chi^{2} / \text { Fisher exact test when necessary. }\end{array}$ & Stroke Scale; mRS = m & ankin Scale; VKA = vit & tagonist. \\
\hline
\end{tabular}

associated with an increased peak PHE volume $>37.495 \mathrm{~mL}$ (median of the entire cohort). In multivariable testing, initial PHE increase up to day 3 and reduced lymphocytes on day 4 remained independent predictors of an increased peak PHE besides a younger age and a higher ICH volume (table 4).

In a propensity score-matched (PSM) subgroup analysis ( $\mathrm{n}=$ 124) accounting for $\mathrm{ICH}$ volume, age, and hematoma location, early PHE increase up to day 3 (OR 1.060 [95\% CI 1.018-1.103]) and increased NLR on day 6 (OR 1.236 [95\% CI 1.034-1.477]) were associated with an increased peak PHE (see also table e-2, links.lww.com/WNL/A259).

\section{Measures associated with early PHE increase up to day 3}

A higher early PHE increase (PSM cohort, $n=224$ ) was significantly associated with a higher fever burden up to day 3 (OR 1.456 [95\% CI 1.103-1.920]) and a hematoma expansion $>5$
$\mathrm{mL}$ up to day 3 (OR 3.647 [95\% CI 1.533-8.679]) while there was no association with age, hematoma volume on admission, or hematoma location, indicating a well-matched cohort. For more detailed information, see table e-3 (links.lww.com/ WNL/A259) (only PSM data shown).

\section{Discussion}

This study suggests that peak PHE volume is an independent predictor of day 90 outcome in ICH patients and is associated with age, hematoma volume, initial PHE increase up to day 3, and inflammatory measures. Each milliliter of additional peak PHE volume increased the absolute risk for a poor outcome by $1.5 \%$. In turn, initial PHE increase was related to hematoma expansion and fever burden. These results may help to direct further research in order to shed light into the pathophysiologic mechanisms that act beyond perihemorrhagic edema evolution 
Table 2 Multivariable logistic regression model modeling the prediction of favorable outcome (modified Rankin Scale $0-3)$ in the intracerebral hemorrhage cohort $(n=292)$

\begin{tabular}{lllr}
\hline & Odds ratio, Exp(B) & 95\% Cl & \multicolumn{1}{c}{ Value } \\
\hline Intraventricular hemorrhage & 0.486 & $0.256-0.887$ & 0.019 \\
\hline NIHSS on admission & 0.936 & $0.906-0.968$ & $<0.001$ \\
\hline Age, $\mathbf{y}$ & 0.944 & $0.918-0.972$ & $<0.001$ \\
\hline Hematoma volume by location, $\mathrm{mL}$ & 0.967 & $0.944-0.990$ & 0.006 \\
\hline Peak perihemorrhagic edema volume, $\mathrm{mL}$ & 0.984 & $0.973-0.994$ & 0.002
\end{tabular}

Abbreviations: $\mathrm{Cl}=$ confidence interval; NIHSS = NIH Stroke Scale.

Hematoma volume by location represents the interaction term hematoma volume $\times$ location with location (basal ganglia vs lobar)-dependent effects of hematoma volume.

and to open up avenues for potential treatment targets in patients with ICH.

After ICH ictus, many inflammatory processes are initiated. ${ }^{1,2}$ Animal studies have revealed a rise of proinflammatory mediators within hours after cerebral injury suggesting a hyperinflammatory state in the initial stages of acute brain injury ${ }^{26}$ associated with the development of brain edema. ${ }^{27}$ Rare human data also suggested an association of edema evolution with inflammatory measures (e.g., $\mathrm{NLR}^{28}{ }^{28}$ certain interleukins, ${ }^{29}$ or matrix metalloproteinase- 9 concentrations ${ }^{30}$ ). Furthermore, the influence of immunomodulating therapies on PHE evolution also supports this association. ${ }^{31,32}$ Those inflammatory processes start within hours after $\mathrm{ICH}$ onset and continue up to several days. ${ }^{1}$ This underlines their importance regarding PHE evolution both in the acute phase and in the postacute period when PHE volume continues to increase. ${ }^{3}$ This link between PHE evolution and inflammatory measures as well as the role of $\mathrm{PHE}$ as an imaging marker of inflammatory processes in $\mathrm{ICH}$ patients is further strengthened and confirmed in light of the findings of our study. It also supports an independent prognostic effect of peak PHE volume on outcome.

Previous reports found a strong correlation between PHE volume and outcome in $\mathrm{ICH}$ patients when analyzing $\mathrm{PHE}$ evolution up to 72 hours after onset. $\mathrm{Wu}$ and colleagues ${ }^{12}$ reported that the early edema extension distance increase up to 72 hours after onset independently influenced mortality after ICH. An analysis by Venkatasubramanian and colleagues $^{33}$ including 27 patients found a correlation of PHE

Figure 1 Evolution of absolute hematoma and perihemorrhagic edema (PHE) volume up to day 10-12 in patients with favorable and poor outcome

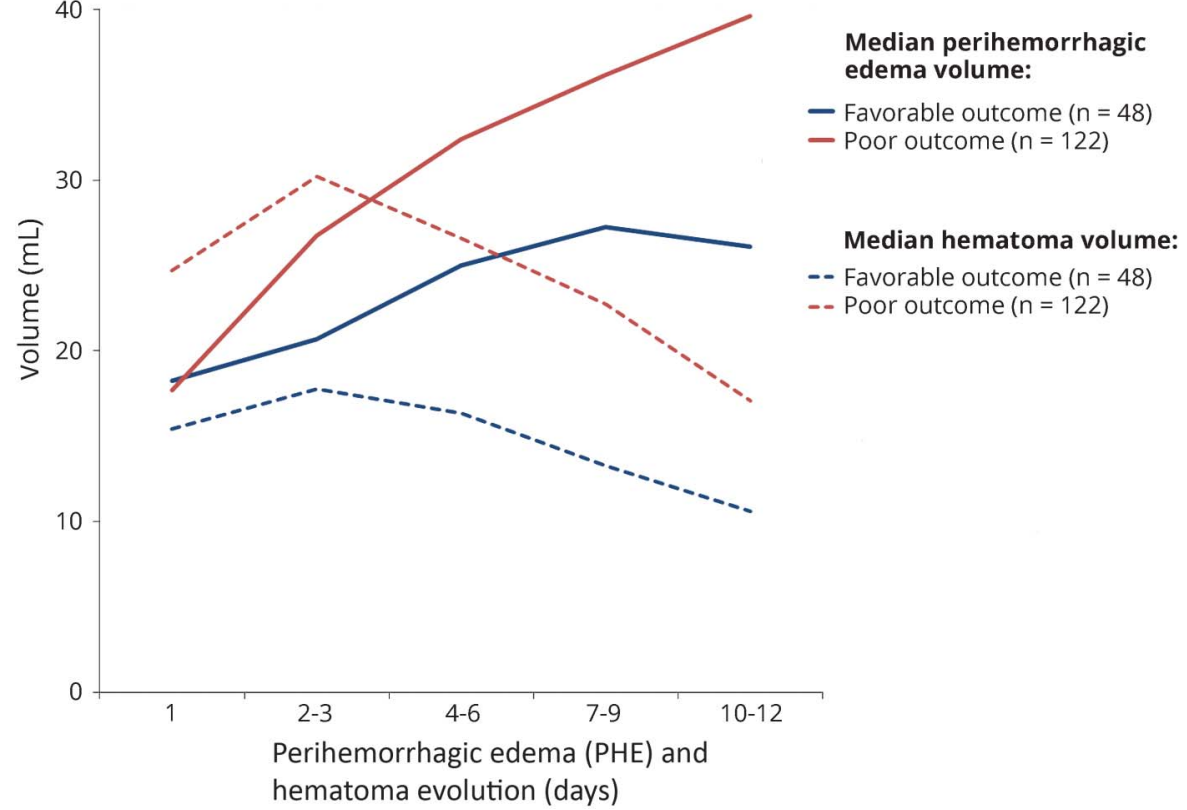

Only patients with $\geq 5$ CT scans up to day 10-12 $(n=170)$ were included. Blue lines represent favorable outcomes, red lines poor outcomes, dotted lines hematoma volumes, and continuous lines perihemorrhagic edema (PHE) volumes. Patients with poor outcome showed not only a higher PHE volume increase during the first 72 hours after onset compared to patients with favorable outcome, but also to a lesser extent up to day $10-12$. 
Table 3 Characteristics of patients with larger and smaller peak perihemorrhagic edema (PHE) volume dichotomized according to median peak PHE volume of the entire cohort (37.495 mL)

\begin{tabular}{|c|c|c|c|}
\hline Spontaneous ICH $(n=292)$ & $\begin{array}{l}\text { Peak PHE volume } \leq 37.495 \mathrm{~mL} \\
(\mathrm{n}=146)\end{array}$ & $\begin{array}{l}\text { Peak PHE volume }>37.495 \mathrm{~mL} \\
(\mathrm{n}=146)\end{array}$ & $p$ Value \\
\hline Age, y, median (IQR) & $71(64-80)$ & $69(61-77)$ & $0.046^{a}$ \\
\hline Male sex, n (\%) & $73(50)$ & $88(60)$ & $0.099^{b}$ \\
\hline Location lobar, $\mathrm{n}(\%)$ & $94(64)$ & $77(53)$ & $0.043^{b}$ \\
\hline Intraventricular hemorrhage, $\mathrm{n}(\%)$ & $71(49)$ & $88(60)$ & $0.046^{\mathrm{b}}$ \\
\hline Hematoma volume on admission, $\mathrm{mL}$, median (IQR) & $8.7(4.2-17.9)$ & $35.0(17.5-53.8)$ & $<0.001^{a}$ \\
\hline Hematoma expansion $>5 \mathrm{~mL}, \mathrm{n}(\%)$ & $9(6)$ & $52(36)$ & $<0.001^{\mathrm{b}}$ \\
\hline PHE volume on admission, mL, median (IQR) & $10.4(5.6-15.1)$ & $28.1(18.4-45.6)$ & $<0.001^{\mathrm{a}}$ \\
\hline PHE volume increase up to day $3, \mathrm{~mL}$, median (IQR) & $2.1(-0.1$ to 5.3$)$ & $8.6(-0.6$ to 17.2$)$ & $<0.001^{\mathrm{a}}$ \\
\hline Fever burden up to day 12, median (IQR) & $14(10-18)$ & $19(12-26)$ & $<0.001^{\mathrm{a}}$ \\
\hline Lymphocytes day $4,10^{9} / \mathrm{L}$, median (IQR) & $1.3(1.0-1.7)$ & $1.1(0.8-1.5)$ & $0.008^{\mathrm{a}}$ \\
\hline Lymphocytes day $6,10^{9} / \mathrm{L}$, median (IQR) & $1.3(0.9-1.6)$ & $1.1(0.8-1.4)$ & $0.006^{a}$ \\
\hline Neutrophil to lymphocyte ratio day 6, median (IQR) & $4.2(3.1-5.6)$ & $4.6(3.2-6.9)$ & $0.031^{a}$ \\
\hline
\end{tabular}

Abbreviations: $\mathrm{ICH}=$ intracerebral hemorrhage; IQR = interquartile range.

${ }^{\text {a }}$ Wilcoxon rank-sum test.

${ }^{\mathrm{b}} \chi^{2} /$ Fisher exact test when necessary.

volume growth up to 48 hours with clinical status at 48 hours while there was no association of total PHE volume growth with 3-month functional outcome. A pooled analysis of the INTERACT data showed that early PHE increase up to 24 hours after onset is an independent predictor of death and dependency on day 90 after ICH. ${ }^{9}$ Similar results could also be shown by Urday et al. ${ }^{11}$ and Murthy et al., ${ }^{34}$ leading to consistent results observed in about 3,000 patients. Our data revealed an unadjusted association of early PHE increase with outcome. Furthermore, as we included PHE data of a wide temporal range, our data suggest that it is primarily the peak PHE volume that matters regarding outcome measures. On the other hand, our analysis also demonstrated an independent correlation of early PHE increase with peak PHE volume. Thus, early PHE increase may serve as a predictor of peak PHE volume. The latter was independently associated with poor outcome in our cohort.

Several therapeutic options targeting the abovementioned mechanisms may evolve. At first sight, removing the blood clot might be the most pragmatic approach. However, so far a general superiority of surgical intervention in ICH patients as compared to conservative treatment could not be shown. ${ }^{35}$ A minimally invasive approach is currently tested in the MISTIE trial, so far yielding promising results concerning PHE reduction in the phase II trial, ${ }^{36}$ however, with an increased rate of asymptomatic bleeding in patients treated with minimally invasive surgery plus alteplase for clot removal. ${ }^{7}$ Hematoma expansion could be limited by administration of a hemostatic therapy with rFVIIa, however, with no

Table 4 Multivariable logistic regression model modeling the prediction of a perihemorrhagic edema (PHE) volume $>37.495 \mathrm{~mL}$ (median PHE volume of the entire intracerebral hemorrhage cohort [ $\mathrm{n}=292]$ )

\begin{tabular}{llll}
\hline & Odds ratio, Exp(B) & $95 \% \mathrm{Cl}$ & $p$ Value \\
\hline Initial PHE volume increase up to day $\mathbf{3}, \mathbf{m L}$ & 1.067 & $1.032-1.104$ & $<0.001$ \\
\hline Hematoma volume on admission, $\mathbf{m L}$ & 1.087 & $1.063-1.112$ & $<0.001$ \\
\hline Hematoma expansion $\mathbf{5} \mathbf{~ m L}$ & 5.749 & $2.304-14.341$ & 0.001 \\
\hline Age, $\mathbf{y}$ & 0.970 & $0.947-0.995$ & 0.017 \\
\hline Lymphocytes on day $\mathbf{4}, \mathbf{1 0} / \mathrm{L}$ & 0.394 & $0.204-0.761$ & 0.006 \\
\hline
\end{tabular}

Abbreviation: $\mathrm{Cl}=$ confidence interval. 
improvement in survival or functional outcome. PHE evolution was not analyzed in this trial. ${ }^{8}$ Another approach targeting multiple pathophysiologic pathways associated with PHE evolution is therapeutic hypothermia. ${ }^{26}$ Therapeutic hypothermia effectively reduced $\mathrm{PHE}$ volume, ${ }^{32}$ especially when initiated early after ICH onset. ${ }^{37}$ These findings underline the importance of early inflammatory processes regarding PHE evolution, as suggested in our study. However, the question how long to treat and which target temperature to obtain to improve outcome needs to be elucidated in future research. A third possible treatment target is inflammation. Only a few studies with small patient numbers analyzed the effect of corticosteroids in ICH patients and found no effect on mortality and infections. ${ }^{38}$ Thus, European Stroke Organisation guidelines do not recommend the use of corticosteroids at this time. ${ }^{39}$ Other anti-inflammatory substances, e. g., fingolimod, are under clinical investigation. ${ }^{31}$ However, it remains to be elucidated whether the inflammatory response related to $\mathrm{PHE}$ evolution represents rather a reaction to the occurrence of blood within brain parenchyma, a reaction to additional infectious diseases in severely ill patients, or both, and whether an immunosuppressive strategy, an antiinfectious strategy, or both will resolve the inflammatory dilemma in $\mathrm{ICH}$ patients.

There are several strengths of the present study: we could include a sound cohort of an adequate size with available longterm data regarding PHE evolution and outcome assessment. Furthermore, a validated measurement was used for quantification of PHE and ICH volume, ${ }^{17}$ yielding reliable and robust results.

There are also several limitations. Despite the proper size of the included cohort, only patients from a single center were included. Due to the retrospective design, there were no standardized time points of imaging. To obtain comparable results, we merged imaging data within prespecified time clusters. This procedure might lead to an underestimation of peak PHE volume and also to a distorted representation of early PHE volume increase up to day 3 and the time axis of PHE evolution. However, this bias exists for all outcome groups. Due to the exclusive inclusion of supratentorial $\mathrm{ICH}$ patients, no statement about the association of PHE with functional outcome in infratentorial $\mathrm{ICH}$ patients is yet possible. A further selection bias also cannot be excluded as we did not include patients with early withdrawal of care and patients with only 1 CT scan $(n=294)$ and excluded 38 patients due to missing data. Because length of hospital stay and number of CT scans varied between individuals, in some patients the real peak PHE volume might not have been assessed. We used the method of median split to assess measures influencing peak PHE volume leading to a possible loss of power in this analysis. Validation of our methodology was performed up to day 5 after admission. We cannot exclude that due to ongoing hematoma degradation a certain inaccuracy may affect our PHE assessment between day 6 and 12 , even if our method corrects for hematoma degradation.
Time between symptom onset and admission did not differ between patients with and without hematoma expansion. However, as this measure differed between individual patients, it might pose possible bias in this complex relation among hematoma evolution, PHE evolution, outcome, and time.

\section{Author contributions}

Bastian Volbers: acquisition, analysis and interpretation of data, drafting the work, final approval, agreement to be accountable for all aspects of the work. Antje Giede-Jeppe: analysis of data, final approval, agreement to be accountable for all aspects of the work. Stefan Gerner: critically revising the work for important intellectual content, final approval, agreement to be accountable for all aspects of the work. Jochen Sembill: critically revising the work for important intellectual content, final approval, agreement to be accountable for all aspects of the work. Joji B. Kuramatsu: interpretation of data, critically revising the work for important intellectual content, final approval, agreement to be accountable for all aspects of the work. Stefan Lang: interpretation of data, critically revising the work for important intellectual content, final approval, agreement to be accountable for all aspects of the work. Hannes Lücking: analysis of data, critically revising the work for important intellectual content, final approval, agreement to be accountable for all aspects of the work. Dimitre Staykov: critically revising the work for important intellectual content, final approval, agreement to be accountable for all aspects of the work. Hagen B. Huttner: analysis and interpretation of data, conceptualization the work, final approval, agreement to be accountable for all aspects of the work.

\section{Study funding}

No targeted funding reported.

\section{Disclosure}

The authors report no disclosures relevant to the manuscript. Go to Neurology.org/N for full disclosures.

Received July 11, 2017. Accepted in final form December 18, 2017.

\section{References}

1. Qureshi AI, Mendelow AD, Hanley DF. Intracerebral haemorrhage. Lancet 2009;373: $1632-1644$.

2. Xi G, Keep RF, Hoff JT. Mechanisms of brain injury after intracerebral haemorrhage. Lancet Neurol 2006;5:53-63.

3. Staykov D, Wagner I, Volbers B, et al. Natural course of perihemorrhagic edema after intracerebral hemorrhage. Stroke 2011;42:2625-2629.

4. Ziai WC. Hematology and inflammatory signaling of intracerebral hemorrhage. Stroke 2013;44:S74-S78.

5. Mayer SA, Sacco RL, Shi T, Mohr JP. Neurologic deterioration in noncomatose patients with supratentorial intracerebral hemorrhage. Neurology 1994;44: 1379-1384.

6. Hanley DF, Lane K, McBee N, et al. Thrombolytic removal of intraventricular haemorrhage in treatment of severe stroke: results of the randomised, multicentre, multiregion, placebo-controlled CLEAR III trial. Lancet 2017;389:603-611.

7. Hanley DF, Thompson RE, Muschelli J, et al. Safety and efficacy of minimally invasive surgery plus alteplase in intracerebral haemorrhage evacuation (MISTIE): a randomised, controlled, open-label, phase 2 trial. Lancet Neurol 2016;15:1228-1237.

8. Mayer SA, Brun NC, Begtrup K, et al. Efficacy and safety of recombinant activated factor VII for acute intracerebral hemorrhage. N Engl J Med 2008;358: 2127-2137. 
9. Yang J, Arima H, Wu G, et al. Prognostic significance of perihematomal edema in acute intracerebral hemorrhage: pooled analysis from the intensive blood pressure reduction in acute cerebral hemorrhage trial studies. Stroke 2015;46:1009-1013.

10. Murthy SB, Urday S, Beslow LA, et al. Rate of perihaematomal oedema expansion is associated with poor clinical outcomes in intracerebral haemorrhage. J Neurol Neurosurg Psychiatry 2016;87:1169-1173.

11. Urday S, Beslow LA, Dai F, et al. Rate of perihematomal edema expansion predicts outcome after intracerebral hemorrhage. Crit Care Med 2016;44:790-797.

12. Wu TY, Sharma G, Strbian D, et al. Natural history of perihematomal edema and impact on outcome after intracerebral hemorrhage. Stroke 2017;48:873-879.

13. Schwarz S, Hafner K, Aschoff A, Schwab S. Incidence and prognostic significance of fever following intracerebral hemorrhage. Neurology 2000;54:354-361.

14. Giede-Jeppe A, Bobinger T, Gerner ST, et al. Lymphocytopenia is an independent predictor of unfavorable functional outcome in spontaneous intracerebral hemorrhage. Stroke 2016;47:1239-1246.

15. Lattanzi S, Cagnetti C, Provinciali L, Silvestrini M. Neutrophil-to-Lymphocyte ratio predicts the outcome of acute intracerebral hemorrhage. Stroke 2016;47:1654-1657.

16. Brouwers HB, Chang Y, Falcone GJ, et al. Predicting hematoma expansion after primary intracerebral hemorrhage. JAMA Neurol 2014;71:158-164.

17. Volbers B, Staykov D, Wagner I, et al. Semi-automatic volumetric assessment of perihemorrhagic edema with computed tomography. Eur J Neurol 2011;18:1323-1328.

18. Grunwald Z, Beslow LA, Urday S, et al. Perihematomal edema expansion rates and patient outcomes in deep and lobar intracerebral hemorrhage. Neurocrit Care 2017; 26:205-212.

19. Broderick JP, Brott TG, Duldner JE, Tomsick T, Huster G. Volume of intracerebral hemorrhage: a powerful and easy-to-use predictor of 30-day mortality. Stroke 1993; 24:987-993.

20. Ziai W, Carhuapoma JR, Nyquist P, Hanley DF. Surgical strategies for spontaneous intracerebral hemorrhage. Semin Neurol 2016;36:531-541.

21. Webb AJ, Ullman NL, Morgan TC, et al. Accuracy of the ABC/2 score for intracerebral hemorrhage: systematic review and analysis of MISTIE, CLEAR-IVH, and CLEAR-III. Stroke 2015;46:2470-2476.

22. Kuramatsu JB, Bobinger T, Volbers B, et al. Hyponatremia is an independent predictor of in-hospital mortality in spontaneous intracerebral hemorrhage. Stroke 2014; 45:1285-1291.

23. Delcourt C, Sato S, Zhang S, et al. Intracerebral hemorrhage location and outcome among INTERACT2 participants. Neurology 2017;88:1408-1414.

24. Sembill JA, Gerner ST, Volbers B, et al. Severity assessment in maximally treated ICH patients: the max-ICH score. Neurology 2017;89:423-431.
25. Hemphill JC III, Bonovich DC, Besmertis L, Manley GT, Johnston SC. The ICH score: a simple, reliable grading scale for intracerebral hemorrhage. Stroke 2001;32: 891-897.

26. Polderman KH. Mechanisms of action, physiological effects, and complications of hypothermia. Crit Care Med 2009;37:S186-S202.

27. Chen S, Yang $Q$, Chen G, Zhang JH. An update on inflammation in the acute phase of intracerebral hemorrhage. Transl Stroke Res 2015;6:4-8.

28. Gusdon AM, Gialdini G, Kone G, et al. Neutrophil-lymphocyte ratio and perihematomal edema growth in intracerebral hemorrhage. Stroke 2017;48:2589-2592.

29. Wang XM, Zhang YG, Li AL, et al. Expressions of serum inflammatory cytokines and their relationship with cerebral edema in patients with acute basal ganglia hemorrhage. Eur Rev Med Pharmacol Sci 2016;20:2868-2871.

30. Abilleira S, Montaner J, Molina CA, Monasterio J, Castillo J, Alvarez-Sabin J. Matrix metalloproteinase- 9 concentration after spontaneous intracerebral hemorrhage. J Neurosurg 2003;99:65-70.

31. Fu Y, Hao J, Zhang N, et al. Fingolimod for the treatment of intracerebral hemorrhage: a 2-arm proof-of-concept study. JAMA Neurol 2014;71:1092-1101.

32. Staykov D, Wagner I, Volbers B, Doerfler A, Schwab S, Kollmar R. Mild prolonged hypothermia for large intracerebral hemorrhage. Neurocrit Care 2013;18:178-183.

33. Venkatasubramanian C, Mlynash M, Finley-Caulfield A, et al. Natural history of perihematomal edema after intracerebral hemorrhage measured by serial magnetic resonance imaging. Stroke 2011;42:73-80.

34. Murthy SB, Moradiya Y, Dawson J, et al. Perihematomal edema and functional outcomes in intracerebral hemorrhage: influence of hematoma volume and location. Stroke 2015;46:3088-3092.

35. Mendelow $\mathrm{AD}$, Gregson $\mathrm{BA}$, Rowan EN, et al. Early surgery versus initial conservative treatment in patients with spontaneous supratentorial lobar intracerebral haematomas (STICH II): a randomised trial. Lancet 2013;382:397-408.

36. Mould WA, Carhuapoma JR, Muschelli J, et al. Minimally invasive surgery plus recombinant tissue-type plasminogen activator for intracerebral hemorrhage evacuation decreases perihematomal edema. Stroke 2013;44:627-634.

37. Volbers B, Herrmann S, Willfarth W, et al. Impact of hypothermia initiation and duration on perihemorrhagic edema evolution after intracerebral hemorrhage. Stroke 2016;47:2249-2255.

38. Feigin VL, Anderson N, Rinkel GJ, Algra A, van Gijn J, Bennett DA. Corticosteroids for aneurysmal subarachnoid haemorrhage and primary intracerebral haemorrhage. Cochrane Database Syst Rev 2005:CD004583.

39. Steiner T, Al-Shahi Salman R, Ntaios G. The European Stroke Organisation (ESO) guidelines. Int J Stroke 2014;9:838-839. 


\section{Peak perihemorrhagic edema correlates with functional outcome in intracerebral hemorrhage}

Bastian Volbers, MD, Antje Giede-Jeppe, MD, Stefan T. Gerner, MD, Jochen A. Sembill, MD, Joji B. Kuramatsu, MD, Stefan Lang, MD, Hannes Lücking, MD, Dimitre Staykov, MD, and Hagen B. Huttner, MD, PhD

Cite as: Neurology ${ }^{\circledR}$ 2018;90:e1005-e1012. doi:10.1212/WNL.0000000000005167
Correspondence

Dr. Volbers

bastian.volbers@gmx.net

\section{Study question}

Are peak perihemorrhagic edema (PHE) volumes associated with functional outcomes 90 days after intracerebral hemorrhage $(\mathrm{ICH})$ ?

\section{Summary answer}

Greater PHE volumes are associated with worse 90-day postICH functional outcomes.

\section{What is known and what this paper adds}

PHE volumes peak within approximately $8-12$ days of the ictus, and this growth is thought to induce clinical deterioration. This study provides evidence that peak PHE volumes are in fact predictors of long-term post-ICH functional outcomes.

\section{Participants and setting}

This study examined 292 patients (55\% male) with spontaneous supratentorial ICH who were admitted to the Neurological Department of the University of Erlangen-Nuremberg between January 2006 and January 2014. All had a baseline modified Rankin Scale (mRS) score of $<4$.

\section{Design, size, and duration}

The study reviewed CT scans in patient medical records to determine peak PHE volumes observed mainly within 12 days of admission. Patient functionality was assessed based on mRS scores.

\section{Primary outcomes}

The primary outcome was the 90-day post-ICH mRS score, which was determined via mailed questionnaires or telephone interviews with patients or their next of kin. Scores of 0-3 were classified as favorable outcomes, and scores of 4-6 were classified as poor outcomes.

\section{Main results and the role of chance}

Favorable 90-day outcomes were recorded for 107 (37\%) patients, whereas unfavorable outcomes were recorded for 185 (63\%) patients. The peak PHE volumes were smaller for patients with favorable outcomes than for patients with unfavorable outcomes (medians [interquartile ranges], 23.8 [9-45.3] mL vs 42.6 [28.1-67.4] mL; $p<0.001)$. Multivariable

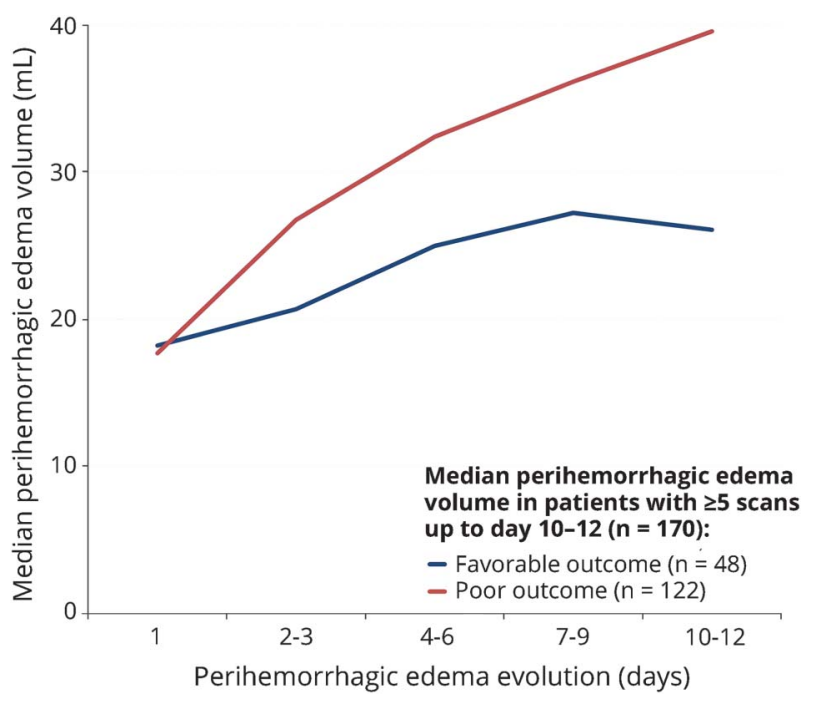

logistic regression confirmed that greater peak PHE volumes reduced the chances of a favorable outcome (odds ratio, 0.984 per $\mathrm{mL} ; 95 \% \mathrm{CI}, 0.973-0.994 ; p=0.002$ ). A receiver operating characteristics analysis showed that a $26.8-\mathrm{mL}$ cut-off value for the peak PHE volume predicted a poor outcome with 54\% sensitivity and $78 \%$ specificity.

\section{Bias, confounding, and other reasons for caution}

This study examined patients at only one center. Furthermore, the imaging timepoints were not standardized, and the exclusion of patients with insufficient CT scans for determination of peak PHE volumes might have introduced selection bias.

\section{Generalizability to other populations}

This study exclusively examined patients with supratentorial $\mathrm{ICH}$, so the results may not be generalizable to patients with infratentorial ICH.

\section{Study funding/potential competing interests}

This study received no funding, and the authors report no competing interests. Go to Neurology.org/N for full disclosures.

A draft of the short-form article was written by M. Dalefield, a writer with Editage, a division of Cactus Communications. The authors of the full-length article and the journal editors edited and approved the final version. 


\section{Neurology}

\section{Peak perihemorrhagic edema correlates with functional outcome in intracerebral hemorrhage \\ Bastian Volbers, Antje Giede-Jeppe, Stefan T. Gerner, et al.}

Neurology 2018;90;e1005-e1012 Published Online before print February 16, 2018

DOI 10.1212/WNL.0000000000005167

This information is current as of February 16, 2018

\section{Updated Information \&} Services

References

Subspecialty Collections

Permissions \& Licensing

Reprints including high resolution figures, can be found at: http://n.neurology.org/content/90/12/e1005.full.html

This article cites 38 articles, 20 of which you can access for free at: http://n.neurology.org/content/90/12/e1005.full.html\#\#ref-list-1

This article, along with others on similar topics, appears in the following collection(s):

All Clinical Neurology

http://n.neurology.org//cgi/collection/all_clinical_neurology

CT

http://n.neurology.org//cgi/collection/ct

Intracerebral hemorrhage

http://n.neurology.org//cgi/collection/intracerebral_hemorrhage

Information about reproducing this article in parts (figures,tables) or in its entirety can be found online at:

http://n.neurology.org/misc/about.xhtml\#permissions

Information about ordering reprints can be found online:

http://n.neurology.org/misc/addir.xhtml\#reprintsus

Neurology ${ }^{\circledR}$ is the official journal of the American Academy of Neurology. Published continuously since 1951, it is now a weekly with 48 issues per year. Copyright () 2018 American Academy of Neurology. All rights reserved. Print ISSN: 0028-3878. Online ISSN: 1526-632X.

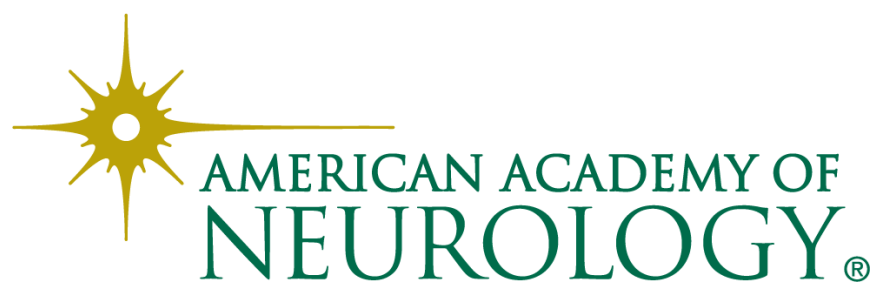

